\title{
VIII. Les collectivités locales à l'épreuve des risques
}

\section{environnementaux}

François Duchene, Emmanuel Martinais

\section{Citer ce document / Cite this document :}

Duchene François, Martinais Emmanuel. VIII. Les collectivités locales à l'épreuve des risques environnementaux. In: Annuaire des collectivités locales. Tome 22, 2002. L'organisation territoriale de la France, demain. pp. 129-148;

doi : 10.3406/coloc.2002.1435

http://www.persee.fr/doc/coloc_0291-4700_2002_num_22_1_1435

Document généré le 03/06/2016 


\title{
VIII. LES COLLECTIVITÉS LOCALES À L'ÉPREUVE DES RISQUES ENVIRONNEMENTAUX
}

\author{
François DuchÊNE, Emmanuel MARTINAIS
}

Depuis quelques années, la problématique du risque, et conjointement celle de la sécurité, occupent une place de plus en plus importante dans le débat public. Qu'il soit d'origine naturelle, industrielle, sanitaire ou même « sociale », le risque traverse désormais la plupart des champs sociaux (politique, administratif, associatif, juridique, économique, assurantiel, médiatique, scientifique, technique, etc.), et tend à s'imposer comme un sujet de préoccupation incontournable pour les populations exposées comme pour bon nombre d'institutions relevant de domaines de compétence divers. Parmi les nombreux acteurs concernés de près ou de loin par cette question, les collectivités locales bénéficient d'une position particulière puisqu'elles doivent généralement concilier leurs impératifs de gestion et de développement avec les contraintes diverses que suppose aujourd'hui la prise en compte des risques au plan local (information du public, réalisation d'ouvrages de protection, limitation de l'urbanisation, organisation des secours, etc.).

Partant de ce constat, il nous paraît intéressant de rendre compte des modalités selon lesquelles les collectivités locales appréhendent, anticipent et traitent les dangers auxquels elles se pensent confrontées. Quels sont les enjeux et les contraintes qui s'imposent à elles ? À quels types d'obstacles sont-elles confrontées ? Dans quelle mesure contribuent-elles à déterminer la nature des solutions adoptées pour faire face aux conséquences prévisibles des phénomènes redoutés ? Les échelles sur lesquelles ces mêmes phénomènes se déploient ont-elles une incidence à ce niveau ? Peut-on faire le constat d'une certaine régularité des modes de faire dans ce domaine ou, au contraire, chaque contexte est-il spécifique au point de déterminer des réponses adaptées à chaque cas d'espèce ? Autant de questions auxquelles nous souhaitons apporter quelques éléments de réponse. Pour ce faire, nous envisageons d'élargir au maximum notre perspective afin de ne pas occulter l'importance de dynamiques 
(sociales ou spatiales) se déployant sur le temps long. Mobiliser ainsi l'histoire permet notamment d'aller chercher dans le passé tout ce qui peut servir à la compréhension du présent, ce qui lui donne du sens, et, au-delà, de construire les bases d'une réflexion prospective en repérant les cadres structurants susceptibles de se reproduire dans l'avenir'.

Dans un premier temps, nous nous intéresserons aux aspects réglementaires encadrant la gestion des risques environnementaux, aux compétences des collectivités locales et à leurs évolutions depuis un siècle environ. Nous montrerons à cette occasion que le seul point de vue législatif est sans doute trop partiel pour appréhender la manière dont le risque est pris en compte au niveau local. En effet, le risque et sa gestion, qui s'inscrivent généralement dans des lieux relativement circonscrits, sont aussi les produits d'une histoire particulière. Le second temps de l'article permettra donc de montrer qu'il n'y a pas de risque qui soit totalement compréhensible en dehors du (ou des) contexte(s) dans lesquels il est susceptible de prendre forme et de l'histoire dans laquelle il s'intègre. Nous verrons, dans un troisième temps, comment les modalités de désignation et de traitement des dangers ne sont jamais vraiment dissociables de l'ensemble des dynamiques territoriales à l'œuvre. Le risque et les solutions élaborées pour y faire face sont toujours le fruit de négociations complexes entre des logiques et des intérêts divergents, parfois même conflictuels, qui n'ont de sens que rapportés au contexte dont il est question et aux enjeux du moment. Pour conclure, nous poserons la question de l'intercommunalité et de ses développements actuels et de la façon dont elle conduit (ou pas) à repenser les modes de gestion du risque, au plan communal comme au niveau supracommunal.

\section{I) Risques, réglementation et collectivités locales}

Obéissant le plus souvent à des logiques sectorielles, les principaux textes réglementaires encadrant la gestion des risques ont relevé pendant longtemps de législations différentes, jusqu'à ce que la prévention et la lutte contre les risques deviennent l'affaire de l'État avec l'avènement d'une politique « globale » dans le courant des années 80 et l'apparition de textes portant à la fois sur les aléas d'origine naturelle et technologique (à l'exception du nucléaire qui fait toujours l'objet d'un traitement à

1. Notre propos sera ponctuellement illustré par des exemples tirés des recherches que nous avons menées sur des terrains concernés soit par des risques dits naturels (mouvements de terrains, inondations, etc.), soit par des risques relevant de l'industrie (chimie, nucleaire, etc.). Pour l'essentiel, ces terrains sont situés dans la grande région lyonnaise : Lyon et son agglomération (couloir de la chimie, balmes, ruisseaux de l'Yzeron et du Ravin, etc.), l'agglomération de Roussillon-Le Péage dans l'Isère (usines Rhodia, centrale nucléaire de Saint-Maurice-l'Exil), la plaine de l'Ain (centrale nucléaire du Bugey). 
part ${ }^{2}$ ). S'agissant de la répartition des compétences en la matière et de leurs évolutions, il convient donc de distinguer deux périodes - avant et après les années 80 - et deux domaines spécifiques : le domaine des risques dits «naturels » (inondations, mouvements de terrains, avalanches, etc.) et celui des risques dits « technologiques », en lien avec les activités industrielles dangereuses (chimie, raffinage, barrages, etc.).

\section{1) La législation sur les risques jusque dans les années 80 : des compétences très variables pour les communes}

À partir de la fin du XIX ${ }^{\mathrm{e}}$ siècle, la prise en compte des aléas d'origine naturelle incombe exclusivement aux municipalités et à leurs représentants élus. Par l'intermédiaire d'une disposition du Code des communes datant de 1884, le droit public prévoit en effet qu'il revient au maire (et à lui seul) « le soin de prévenir, par des précautions convenables, et de faire cesser, par la distribution des secours nécessaires, les accidents et fléaux calamiteux tels que les inondations, les ruptures de digues, les éboulements de terre et de rochers, les avalanches et autres accidents naturels [...], de pourvoir d'urgence à toutes les mesures d'assistance et de secours ${ }^{3} \gg$. La reconnaissance d'un danger de telle nature place donc le représentant de l'autorité municipale dans l'obligation de prescrire les mesures de protection nécessaires au rétablissement de la sécurité publique. Dans une perspective de préservation de l'ordre public et en vertu de ces pouvoirs régaliens, conférés par les règlements de police générale, le maire est alors le seul gestionnaire responsable de la sauvegarde des biens et des personnes sur le territoire de sa commune ${ }^{4}$.

Ces dispositions réglementaires d'ordre général, très inégalement suivies d'une commune à l'autre au cours de la première moitié du $\mathrm{xx}^{\mathrm{e}}$ siècle, connaissent cependant quelques modifications avec l'apparition du Code de l'urbanisme en 1955 et la possibilité de réglementer ou d'interdire les constructions dans des secteurs préalablement identifiés comme dangereux (articles R. 111-2 et R. 111-3). En particulier, l'article 2 du règlement national d'urbanisme prévoit la délimitation par arrêté préfec-

2. Dans les documents préparatoires à la loi du 22 juillet 1987, plusieurs raisons sont invoquées. Tout d'abord le nucléaire est une industrie récente, contrairement à la chimie, qui aurait ainsi réellement pu intégrer en amont les questions relevant de la sécurité ; ensuite, il s'agit d'une industrie située, en France, hors du secteur compétitif, et où des moyens extrêmement importants ont pu être consacrés à la sécurité ; enfin, compte tenu du caractère prévisible et progressif $d$ 'un accident nucléaire, la limitation urbaine autour des centrales ne s'imposerait pas, le temps étant laissé aux autorités d'organiser le confinement, voire l'évacuation des zones potentiellement contaminées en cas d'accident. Au-delà de ces raisons « officielles », on peut surtout rappeler les origines militaires de l'industrie nucléaire en France, dont certaines caractéristiques perdurent largement, au moins à travers un certain goût du secret.

3. Article L. 131-2 du Code des communes. Ces dispositions furent d'ailleurs confirmées par la loi du 21 juin 1898 sur la salubrité publique (article 7).

4. C'est toujours le cas aujourd'hui, ainsi que le stipule le Code général des collectivités territoriales (article L. $2212-2,5^{\circ}$ ). 
toral de zones dans lesquelles la délivrance des permis de construire peut être refusée du fait de l'existence avérée d'un risque naturel. Cette évolution, qui élargit sensiblement le cadre d'intervention des municipalités, consacre aussi l'apparition de la responsabilité de l'État en la matière, sans que celle de la commune soit dégagée pour autant $^{5}$. L'arsenal réglementaire est encore renforcé avec la loi d'orientation foncière (LOF) de 1967 qui ouvre l'ère des documents d'urbanisme et introduit la possibilité de définir des zones $\mathrm{Nd}$ pour cause d'exposition à des aléas d'origine naturelle. De la réalisation d'ouvrages de protection aux restrictions imposées à la construction dans certains secteurs, l'éventail des mesures offertes aux communes pour lutter contre les risques naturels est donc déjà relativement important.

Il en va tout autrement dans le domaine des risques industriels où deux grands principes partiellement antagonistes ont continuellement structuré 1'action du législateur : la liberté économique des industriels d'une part, et d'autre part, la protection de la propriété privée dans le voisinage des établissements dangereux, insalubres ou incommodes. Les différents textes législatifs (décret impérial de 1810, lois de 1917 et de 1976 relatives aux installations classées pour la protection de l'environnement) se situent toujours sur cet axe, sans jamais abandonner entièrement l'un des deux termes. Bien que présenté comme une réponse aux demandes des citadins souffrant du voisinage des établissements nuisants, le premier d'entre eux (décret impérial du 15 octobre 1810 relatif aux manufactures et ateliers insalubres, incommodes ou dangereux) est en fait davantage d'inspiration industrialiste, dans la mesure où il est d'abord pensé comme un moyen de favoriser l'essor industriel et d'assurer sa prospérité : «protéger le patron contre la jalousie ou la malveillance du voisinage, assurer sa tranquillité et, de ce fait, permettre l'expansion de son entreprise s'affirment les soucis majeurs » (Corbin, 1991, p. 188). Ce texte constitue donc moins une mesure de santé publique ou de lutte contre les nuisances causées par les usines polluantes qu'un instrument de gestion de l'espace urbain, un moyen d'arbitrage entre les propriétaires d'établissements industriels et les propriétaires fonciers ou immobiliers les entourant (Massard-Guilbaud, 1999).

Le deuxième (loi du 19 décembre 1917), qui concrétise plusieurs tentatives de réforme de la législation sur les établissements classés, est d'abord une réponse aux évolutions des points de vue hygiénistes vis-à-vis de l'industrialisation et à la recon-

5. Comme le montrent très bien les jugements rendus suite à l'avalanche de Val-d'Isère du 10 février 1970 qui fit près de quarante morts. Après que les juges du tribunal administratif de Grenoble eurent déclaré l'État et la commune de Val-d'Isère responsables chacun pour moitié de la catastrophe (jugement du 10 juin 1974), le Conseil d'État, constatant «qu'il n'a pas été procédé de façon approfondie à l'étude des zones exposées à des risques d'avalanche », a finalement décidé que «l'insuffisance de mesures de prévision et de prévention prises par la commune a constitué une faute de nature à engager sa responsabilité vis-à-vis des victimes » et que « la circonstance que le retard apporté à la délimitation des zones exposées aux risques naturels engage la responsabilité de l'État auquel il incombe de prendre les mesures imposées par la législation de l'urbanisme n'est pas de nature à exonérer la commune de la responsabilité qu'elle encourt du fait de ses obligations en matière de police de la sécurité » (jugement du 14 mars 1986). 
naissance des dangers exportés par l'usine. Cette nouvelle loi prétend désormais s'attaquer aux «causes de danger ou inconvénients, soit pour la sécurité, la salubrité ou la commodité du voisinage, soit pour la santé publique, soit encore pour l'agriculture $^{6} »$. Mais en dépit de ce changement d'objet, le texte entend surtout créer un système encore plus libéral, permettant de protéger le développement industriel par l'allégement du contrôle administratif (pourtant resté quasi théorique jusque-là). Quant à la loi de 1976, elle apporte un certain nombre de modifications au régime général en vigueur jusque-là. En particulier, les intérêts de la collectivité protégés par la loi sont considérablement élargis, passant de la «protection du voisinage » à celle de la « nature » et de « l'environnement » au sens large. Dans le même temps, tous les établissements potentiellement dangereux ou polluants sont désormais visés (publics et privés, chimiques, pétrochimiques, agricoles, etc.).

Mais en dépit de ces évolutions notables, le principe général du dispositif réglementaire encadrant les activités industrielles est resté globalement inchangé : répartis en deux ou trois classes et soumis à un régime de déclaration ou d'autorisation (en fonction de la classe et de la dangerosité présumée des produits utilisés ou fabriqués), les installations classées n'ont pas d'interdiction formelle de polluer. En outre, l'autorisation délivrée par arrêté préfectoral spécifiant l'ensemble des mesures susceptibles de pallier les dangers et les inconvénients des installations en cause, doit toujours prendre en compte les capacités techniques et financières dont dispose l'industriel demandeur. Pour l'essentiel, les principales régulations en matière de sécurité s'effectuent donc dans le cadre de ces procédures d'autorisation, en fonction des marges de manœuvre affichées par l'industriel au moment de sa demande. Et si ce type de procédure implique en théorie les représentants d'un grand nombre d'institutions (dont certaines, comme les communes, supposées être garantes du respect des droits du voisinage), dans les faits l'intervention de la plupart d'entre eux reste purement formelle. En réalité, seuls trois acteurs y jouent un véritable rôle : l'industriel à l'origine du projet, le service de l'inspection des établissements classés chargé de définir les prescriptions techniques et le préfet qui délivre ou non l'autorisation.

Dans ce schéma, si les municipalités sont systématiquement consultées et libres d'exprimer leur point de vue (sur l'opportunité d'une nouvelle implantation ou d'une extension d'une usine existante par exemple), il apparaît que ce dernier est rarement pris en compte. Il est même constamment ignoré après l'adoption de la loi de 1917, qui ne laisse plus de place aux revendications du voisinage des usines chimiques et de leurs représentants élus soucieux de préserver la qualité du cadre de vie de leur commune. De la même manière, dans la période qui suit la Seconde Guerre mondiale, le rôle consultatif des collectivités locales reste purement formel, les considérations relatives à l'hygiène et à la sécurité n'étant que de peu de poids face aux perspectives de développement industriel. Durant plusieurs décennies, le manque de

6. Article $1^{\text {er }}$ de la loi du 19 décembre 1917 relative aux établissements dangereux, insalubres ou incommodes. 
conviction ou la rareté des sollicitations extérieures, le contexte réglementaire peu répressif, l'absence de moyens et l'indulgence des instances chargées de veiller au respect des exigences élémentaires en matière d'hygiène et de sécurité, ont donc tendance à confiner la problématique du risque industriel dans un champ de relations sociales qui ne débordent pas, pratiquement, l'enceinte des usines. Il faut en fait attendre les années 80 pour constater une évolution sensible des rapports de force en présence et une plus grande implication des collectivités locales dans la lutte contre les dangers d'origine industrielle.

\section{2) Le tournant des années 80 : la politique des « risques majeurs »}

Depuis le milieu des années 70 , un certain nombre d'évolutions au sein du champ juridico-administratif conduisent au report progressif sur l'État de l'essentiel des prérogatives dans le domaine de la prévention des risques industriels et naturels. Les modifications apportées à la police des installations classées par la loi du 19 juillet 1976, déjà évoquée, annonçaient déjà cette tendance en donnant les pleins pouvoirs à l'administration locale en matière de suivi des activités industrielles dangereuses (Lascoumes, 1989). S'agissant des risques naturels, la situation n'est guère différente, puisque, en 1984, le décret d'application de la loi du 13 juillet $1982^{7}$ confie aux services de l'État le soin d'élaborer les plans d'expositions aux risques (PER), supposés réglementer l'usage des sols dans les secteurs exposés à divers types de dangers (inondations, avalanches, mouvements de terrains, etc.).

Dans le même temps, d'importantes transformations s'opèrent au niveau des administrations centrales, visant à renforcer à la fois leurs compétences et leurs capacités d'expertise. La loi de 1976 s'inscrivait déjà dans le prolongement de l'attribution des missions d'inspection des établissements classés au corps des Mines et, de façon liée, de la création du Service de l'environnement industriel (SEI) au sein du ministère de l'Environnement. D'une façon comparable, la loi du 13 juillet 1982 qui,

7. Répondant pour une large part à l'émotion soulevée par les inondations spectaculaires de l'hiver 1981-1982 (bassins de la Saône, du Rhône et de la Garonne) ainsi qu'au caractère insuffisant, sinon injuste, des réparations auxquelles elles ouvraient droit, et faisant suite à une négociation entre l'État et les assureurs portant sur la modification des régimes existants (Vié le Sage, 1989), la loi du 13 juillet 1982 relative $\grave{a}$ « l'indemnisation des victimes de catastrophes naturelles » est supposée garantir la réparation « des dommages matériels directs ayant eu pour cause déterminante l'intensité anormale d'un agent naturel, lorsque les mesures habituelles à prendre pour prévenir ces dommages n'ont pu empêcher leur survenance ou n'ont pu être prises » (article $1^{\mathrm{er}}$ ). Prévues par cette loi, les dispositions relatives à l'élaboration des PER sont néanmoins précisées par le décret du 3 mai 1984. Elles stipulent notamment que les documents préparés par le service extérieur de l'État chargé d'instruire le projet délimiteront à l'intérieur du périmètre du plan: 1) une zone «rouge » estimée très exposée qui sera rendue inconstructible ; 2) une zone « bleue » exposée à des risques moindres ; 3) une zone « blanche » sans risque prévisible (article 5). 
pour l'essentiel correspond à une extension de la logique assurantielle au domaine des risques naturels, est indissociable de la création d'une Délégation aux risques majeurs, d'abord autonome puis rattachée et intégrée au ministère de l'Environnement (Gilbert, 1995).

Ce faisant, la prévention et la lutte contre les risques, qu'ils soient d'origine industrielle ou naturelle, s'imposent dans le courant des années 80 comme « l'affaire de l'État » (Caillosse, 1990). Plus qu'une simple réponse aux quelques catastrophes survenues dans la décennie précédente, certains voient dans ce renforcement significatif des prérogatives régaliennes en matière de sécurité, une tentative de redonner sens et cohérence à des phénomènes qui, par leur ampleur et leur diversité, tendent à échapper aux schémas d'action classiques de la puissance publique, dans un contexte marqué par la montée en force de "l'économie sociale de marché » comme norme dominante et la poussée dérégulationniste qui vise à substituer, un peu partout en Europe, les mécanismes du marché aux procédures administratives jugées trop contraignantes (Lemieux et Barthe, 1998). D'autres interprètent cette « re-centralisation du pouvoir de police » comme une volonté de redistribuer certaines cartes de la décentralisation : l'État, en position défensive sur beaucoup de grands dossiers impliquant les collectivités locales, en particulier ceux liés à la maîtrise des sols, semblerait avoir trouvé là un point d'appui relativement solide, lui permettant de réaffirmer certaines de ses prérogatives (Marion et Prétot, 1987).

Quoi qu'il en soit, ces évolutions témoignent de l'émergence au sein du champ technico-administratif d'un nouvel objet, le « risque majeur ${ }^{8}$ », défini et validé par des institutions et des systèmes de légitimation assez puissants pour fonder une catégorie mentale spécifique, de portée collective, et au-delà, des pratiques (techniques, normatives, administratives) et des modes d'appréhension ou d'évaluation particuliers. Le « risque majeur », qui semble à lui seul justifier le renouvellement des dispositifs de prévention, trouve d'ailleurs sa consécration juridique avec la loi du 22 juillet 1987, relative à « l'organisation de la sécurité civile, à la protection de la forêt contre l'incendie et à la prévention des risques majeurs ». Le ministère de l'Environnement, très actif dans la conception et l'élaboration de ce texte, s'est aussi chargé de la mise en cuvre de ses deux principales orientations : l'information du public et la traduction du risque dans les documents d'urbanisme. En particulier, le chapitre II de la loi, intitulé « maîtrise de l'urbanisation », modifie les dispositions en vigueur du Code de l'urbanisme et crée de nouvelles obligations pour les communes, destinées à limiter (voire réduire) la densité urbaine dans les secteurs exposés à certains types de dangers préalablement identifiés par les services compétents de l'État ${ }^{9}$. Les outils cartographiques, déjà partiellement utilisés dans le cadre des procédures type R. 111-3 prévues

8. Pour un aperçu de la construction de cette notion, voir DECROP et GiLBERT, 1993.

9. Du fait de ces dispositions, toujours en vigueur aujourd'hui, il revient à l'État de dire le risque et aux communes de le traduire dans leurs documents d'urbanisme (PLU et SCOT notamment), mais aussi d'informer les populations des dangers qu'elles encourent. 
par le Code de l'urbanisme, puis pour l'élaboration des PER après 1984, ont alors tendance à se généraliser, la « mise en espace » des dangers et les zonages contenus dans les documents d'aménagements exécutoires devenant par la même occasion la «clé de voûte de l'édifice de prévention » (Chaline et Dubois-Maury, 1994).

Mais en dépit de toutes ces dispositions visant à parfaire les modalités de traitement des dangers d'origine industrielle et naturelle ${ }^{10}$, la gestion des risques reste un exercice périlleux, souvent problématique. Dans bien des cas, l'épreuve du terrain révèle de nombreux obstacles, qui se posent comme autant de limites à une application stricte de la loi - quand elle s'applique, ce qui n'est pas toujours le cas. Il n'est donc pas rare que l'écart entre les objectifs affichés par l'État dans ce domaine et les dispositions effectivement retenues localement soit important : « la multiplication des textes [...], l'ambiguïté de la terminologie et la diversité, pour ne pas dire les aspects contradictoires, des instruments montrent bien la difficulté de répondre, à l'échelon national, de façon satisfaisante, aux problèmes tels qu'ils se posent dans la réalité quotidienne de la gestion locale des risques », concluait un récent rapport d'évaluation de la politique de prévention des risques naturels ${ }^{11}$. Par conséquent, si la prévention des risques s'inscrit d'abord dans un cadre fixé par des logiques globales, il semble bien que, dans la pratique, celles-ci doivent nécessairement s'articuler avec une certaine forme de réalitê locale, celle à laquelle les communes doivent faire face dans l'exercice quotidien de leurs compétences. En dépit du fait que leur intervention dans ce domaine est censée répondre aux obligations légales fixées par la loi, il reste que, dans bien des cas, elles éprouvent certaines difficultés à faire jouer les dispositifs préventifs tels qu'ils devraient être mis en œuvre d'une manière théorique.

Cela étant, de nombreux exemples montrent que les dispositions réglementaires en vigueur à un moment donné (aussi restrictives et contraignantes soient-elles) n'empêchent pas certaines collectivités locales de mettre en œuvre des actions particulièrement innovantes ou de contribuer, d'une manière active, à l'élaboration de mesures préventives dotées d'une certaine efficacité. De fait, depuis la fin du $\mathrm{XIX}^{\mathrm{e}}$ siècle, les municipalités ont toujours eu (et ont encore) un rôle moteur à jouer dans les prises de décisions concernant la gestion des risques. C'est vrai à toutes les époques et quelle que soit l'intensité avec laquelle les problèmes se posent. Ainsi, certaines municipalités du couloir de la chimie lyonnais ont-elles régulièrement œuvré pour préserver le cadre de vie des secteurs résidentiels entourant les usines chimiques, en incitant des industriels en général peu soucieux de leur voisinage à modifier leurs usages en matière d'hygiène et de sécurité. De même, la municipalité

10. Ajoutons, pour être totalement exhaustif, la loi du 2 février 1995 relative au « renforcement de la protection de l'environnement », qui donne une assise juridique au principe de précaution. Au-delà, elle traduit la volonté d'avoir un cadre réglementaire unique (plan de prévention des risques ou PPR) en substitution de tous les autres (PSS, périmètres R. 111-3, PER, etc.) et la possibilité de faire jouer l'indemnisation avant la catastrophe avec la création d'un fonds spécifique.

11. Rapport d'évaluation sur la politique de prévention des risques naturels, Comité interministériel de l'évaluation des politiques publiques, Paris, La Documentation française, septembre 1997. 
lyonnaise s'est-elle dotée, en marge des dispositions prévues par la loi, d'un dispositif préventif permettant de lutter efficacement contre l'instabilité des versants urbanisés des collines de Fourvière et de la Croix-Rousse (Martinais, 2001). Autrement dit, les modes d'action des collectivités locales et les choix qu'elles opèrent pour prendre en compte les dangers auxquels elles sont confrontées sont très variables d'une époque à une autre, d'un lieu à un autre. La gestion et la prévention des risques au niveau local sont donc toujours plus ou moins conjoncturels. Dans ce domaine, le contexte s'avère être un facteur déterminant.

\section{II) Les risques et leur gestion : l'importance du contexte}

Contrairement aux définitions usuelles qui associent le risque à la confrontation d'un aléa et d'une ou plusieurs vulnérabilités, nous souhaitons plutôt l'appréhender comme une entité hybride qui s'élabore en permanence selon des logiques individuelles et/ou collectives. Dans cette perspective, le risque peut être défini comme le mode de représentation d'un danger donné, comme la projection d'une catastrophe ou d'un malheur à venir. De fait, le risque est avant tout une image «fabriquée », socialement construite, selon des processus dynamiques impliquant une multitude d'acteurs et d'échelles territoriales. S'il fait l'objet de définitions et de représentations savantes (par le biais de la modélisation et de la cartographie notamment), le risque se construit aussi au travers d'un ensemble de pratiques et des représentations forgées dans le quotidien, d'une perception globale de l'environnement proche (naturel, industriel, urbain, social) et de ses évolutions, et plus largement, de la société locale dans laquelle il s'inscrit.

\section{1) Le risque comme représentation}

De notre point de vue, le risque n'est donc pas (ou pas seulement) une quantité mesurable, objectivable. Il faut plutôt l'envisager comme un fait social éminemment subjectif, relatif et donc évolutif. C'est-à-dire que le risque naît de la perception d'un danger dans un contexte social et historique bien défini. Il ne peut donc être étudié indépendamment des individus et des instances qui s'en saisissent, l'identifient et le «construisent », mais aussi des politiques préventives qui peuvent être élaborées pour le neutraliser ou, à défaut, le minimiser. De sorte que les dangers en eux-mêmes ont moins d'intérêt que la façon dont ils sont représentés, formulés, « pris en charge » et mis en débat au sein des sociétés locales concernées.

Si éprouver un risque, c'est se représenter mentalement ou intellectuellement un danger donné, cela revient à fournir un travail d'interprétation, plus ou moins conscient et formalisé, qui consiste à reconnaître l'existence de ce danger, à en iden- 
tifier les origines probables, les mécanismes physiques par lesquels il se manifeste, puis à envisager les effets qu'il est possible de lui attribuer, ainsi que les conséquences induites pour soi, pour le (ou les) groupes sociaux dans lesquels on s'insère, ou même pour la collectivité à laquelle on appartient. Toutes ces opérations mentales, aussi nécessaires qu'inévitables pour qui se trouve confronté à la perspective du malheur, de la maladie ou de la mort, n'ont qu'un but : il s'agit de donner corps à ce qui n'est pas encore arrivé, de rationaliser l'inconnu, de lui donner du sens, de s'en rendre maître en quelque sorte, pour pouvoir ensuite s'en protéger.

Mais si le risque résulte toujours d'un travail d'interprétation et d'anticipation d'une situation donnée (la proximité d'une usine chimique, d'une rivière, etc.), les modalités de ce travail empruntent autant de chemins qu'il y a d'individus pour se représenter le danger en question. Pour reprendre le cas de la chimie, le risque n'a pas le même contenu et le même sens selon qu'il est appréhendé par un ouvrier posté dans une installation dangereuse, le responsable de fabrication de cette même installation, l'inspecteur chargé de la contrôler, le riverain dont les fenêtres donnent sur l'usine, ou l'élu de la commune concernée.

En réalité, le risque s'inscrit toujours en filigrane de pratiques et de représentations forgées dans le quotidien, dans un rapport privilégié à l'environnement urbain, social, humain ou professionnel. L'expérience sensible (voire sensitive) du cadre de vie ou du milieu professionnel, le vécu et la mémoire des lieux et des événements passés, la perception de l'espace, les préoccupations et les contraintes de la vie quotidienne, la prégnance d'une «culture» (qu'elle soit professionnelle, technique, politique, syndicale, ouvrière, ou même locale), l'expression de sensibilités ou de croyances particulières, les contraintes imposées par un statut social, par un statut résidentiel, la plus ou moins grande réceptivité aux diverses sources d'informations qui circulent (les rumeurs, les témoignages de proches, d'amis, de voisins), et bien d'autres facteurs encore, sont autant d'éléments capables de structurer la relation au danger, dans des proportions qui varient considérablement selon les individus et les groupes sociaux.

Mais l'affaire se complique encore, car un même individu peut avoir recours à diverses formes de représentation d'un même danger. Un ouvrier de la chimie, par exemple, peut très bien avoir des activités syndicales au sein de son entreprise, habiter à proximité de l'usine dans laquelle il travaille, et être membre du conseil municipal. Du coup, il n'appréhendera pas le danger de la même façon, selon qu'il le pense en tant qu'ouvrier, syndicaliste, riverain ou élu. Pour ce même individu, le risque est alors moins le résultat d'une construction monolithique que la formation d'un système de représentations différenciées, plus ou moins cohérentes et plus ou moins conflictuelles.

De plus, et pour rester dans le contexte de la chimie, la plupart des individus auxquels nous nous intéressons (riverains, salariés, syndicalistes, élus, dirigeants industriels, etc.) s'inscrivent la plupart du temps dans un collectif (groupement d'habitants protestataires), un groupe professionnel (manutentionnaires, conducteurs d'appareils, contremaîtres, chimistes, etc.) ou une institution (section syndicale, 
municipalité, entreprise). À ce titre, ils sont aussi en mesure de mobiliser des représentations élaborées collectivement au sein de ces différentes instances, représentations qu'ils subissent en même temps qu'ils les alimentent. De fait, le système de représentations associé à chaque individu comporte plusieurs niveaux, mêlant dans un ensemble d'interactions, plus ou moins complexe selon les cas, des perceptions élaborées individuellement et collectivement.

L'anticipation du danger, d'où qu'elle vienne, s'exprime donc toujours à travers un système plus ou moins cohérent et plus ou moins conflictuel de représentations, liées à des pratiques «ordinaires », professionnelles et institutionnelles. D'une certaine façon, chaque individu, chaque groupe social, chaque institution pratique en permanence ce travail d'anticipation vis-à-vis d'aléas dont la nature, les effets potentiels et les conséquences envisagées peuvent être extrêmement variés. Cette construction peut être plus ou moins collective, plus ou moins identifiable dans un ensemble de pratiques données, plus ou moins formalisée et conceptualisée, plus ou moins consciente, plus ou moins technique, mais elle ne peut pas ne pas exister.

\section{2) Le risque, toujours « en construction », ne peut se définir dans l'absolu}

Une autre caractéristique remarquable du risque, c'est qu'il n'est jamais figé. Plusieurs types de facteurs peuvent entraîner ces transformations d'un système de représentations en un autre. Il s'agit bien évidemment de l'événement accidentel qui, au-delà de ses conséquences matérielles et fonctionnelles, a cette capacité de réactiver la nécessité du sens évoquée précédemment. Mais il peut aussi s'agir de l'absence notable d'accident, sur une période plus ou moins longue, le temps qui passe ayant tendance à relativiser l'importance accordée au danger. D'autre part, la réalisation de travaux de protection ou la mise en place de dispositifs de sécurité sont aussi des éléments susceptibles d'induire une transformation des représentations à l'œuvre, et ce d'autant plus qu'ils sont visibles et font la preuve de leur efficacité. Enfin, l'apport de connaissances sur les phénomènes en cause et les modalités de diffusion de ces connaissances jouent aussi un rôle évident à ce niveau. En particulier, les médias disposent d'une position souvent déterminante en la matière.

Puisque le risque est toujours en train de se deformer et de se reformer, les convictions plus ou moins absolues sur lesquelles se fondent les agents en position de décider (élus, scientifiques, techniciens, responsables administratifs, etc.) sont toujours provisoires. Et cela tient sans doute moins aux incertitudes techniques proprement dites qu'au système de représentations du danger, en vigueur pour chacun de ces agents à un moment donné. En effet, cette sorte d'enveloppe mentale indépassable, cet horizon de référence à l'intérieur duquel s'opère l'évaluation de la situation et la prise de décision, est toujours susceptible d'être revisitée, à mesure que les connaissances progressent ou que les accidents surviennent. Bien souvent, les événements accidentels ou les catastrophes résultent ainsi, non pas d'un défaut d'appréciation ou 
d'interprétation, mais d'une évaluation cohérente en regard de systèmes de représentations qui vont se révéler dépassés. Pour le dire autrement, les signes annonciateurs d'un accident ne sont généralement évidents que pour qui sait les « lire », c'est-à-dire les replacer dans une perspective qui leur donne un sens - mais cette perspective ne prend généralement sens qu'après coup.

Tous les facteurs influents que nous avons préalablement identifiés, lorsqu'ils sont de nature à imposer de nouvelles perceptions du danger, opèrent donc la transformation (selon des modalités propres à chaque individu, chaque groupe social, chaque institution) d'un système de représentations en un autre. Le premier, devenu caduc, laisse la place au nouveau qui devient alors, toujours à titre provisoire, le nouvel horizon de référence. Pour autant, ces transformations ne s'effectuent pas nécessairement dans les mêmes conditions et aux mêmes moments pour l'ensemble des individus concernés, notamment parce que l'information et le savoir ne se transmettent pas dans un espace social homogène et totalement isotrope, mais dans un espace « gauchi » par la nature des relations sociales établies dans la longue durée. De ce fait, la relation qu'une société locale entretient avec un danger donné suppose l'existence d'un nombre illimité d'horizons de référence (autant de définitions du risque qu'il y a d'acteurs pour l'appréhender), qui se distinguent autant par leur nature que par leur temporalité. C'est pourquoi le danger a aussi cette capacité de susciter des réactions particulièrement diversifiées, selon les époques et les acteurs concernés par son existence (Coanus et al., 1999).

Si les actions de prévention et la production de sécurité sont pour partie le reflet des représentations du danger en vigueur à un moment donné (voire des décalages qui subsistent entre eux), d'autres éléments propres au contexte de l'époque sont susceptibles d'orienter les choix, individuels ou collectifs, qui s'opèrent lorsqu'une société locale (ou une partie de celle-ci) est confrontée à la perspective de l'accident ou de la catastrophe. Même si la gestion des risques prend souvent les apparences d'un domaine d'intervention saturé de rationalité (rationalité administrative, juridique, technique, scientifique), il n'en reste pas moins qu'elle est aussi le produit de déterminants sociaux et territoriaux.

\section{III) Le risque, produit de dynamiques territoriales}

À observer les modes de gestion du risque au niveau local, on constate que, bien souvent, plusieurs années s'écoulent entre la prise de conscience de l'existence d'un danger et son traitement. Cette période, généralement attribuée au temps nécessaire pour adapter les dispositions réglementaires ou programmer les montages financiers, correspond au moins autant (sinon davantage) à la durée de résolution des conflits que ne manquent pas de soulever ces traitements. En effet, la question du risque n'est jamais isolée du reste des préoccupations, voire plus profondément des antagonismes, à l'œuvre sur un territoire. Et si une actualité catastrophique (inonda- 
tion, explosion, etc.) vient un temps bousculer les ordres de priorités de l'action locale, les dynamiques territoriales structurantes reprennent bien vite le pas. Pour expliciter notre propos, on distinguera arbitrairement plusieurs types de dynamiques territoriales, en réalité assez étroitement intriqués, susceptibles d'interférer sur la formulation locale de la question du risque.

\section{1) Risque et conflits socio-spatiaux}

Le premier concerne le rapport à l'espace qu'entretiennent les groupes sociaux dominant un territoire. Au-delà de conflits ponctuels, on observe que la question du risque se trouve prise d'une façon plus globale dans les conflits socio-spatiaux qui structurent un territoire sur une période longue. Autrement dit, les solutions envisagées, celles finalement adoptées sont bien le fruit de la prise en compte de préoccupations « globales », certes, mais à chaque fois visitées et le cas échéant corrigées au prisme des intérêts des groupes dominant la société locale où elles s'appliquent, au moment où la question se pose.

Une lecture diachronique des archives du Service hydraulique des ponts et chaussées du Rhône (entre 1851 et 1931) montre par exemple que les plaintes de souillure de la rivière d'Yzeron ${ }^{12}$, exprimées tantôt par les agriculteurs à l'égard des blanchisseurs, tantôt réciproquement ${ }^{13}$, s'inscrivent dans un conflit d'usages ancien autour de la ressource en eau, de la part de deux groupes encore économiquement influents dans les communes urbaines riveraines de ce ruisseau, dans la seconde moitié du XIX ${ }^{e}$ siècle. Et les craintes d'un risque sanitaire lié à ces souillures en régime de basses eaux, préoccupations aux fondements hygiénistes exprimées localement avec force par un notable médecin, ne trouvent finalement de traduction opérationnelle qu'au début du $\mathrm{xx}^{\mathrm{e}}$ siècle, lorsque les deux groupes incriminés tendent à disparaître, les uns du fait de l'industrialisation des communes urbaines situées en aval de la rivière, les autres du fait de la mécanisation du blanchissage (Duchêne, Morel Journel et al., 2001).

Dans le même esprit, nous avons montré, à travers une approche diachronique de l'agglomeration roussillonnaise, en Isère, combien les facteurs d'évolution des représentations du danger sont à rechercher du côté des antagonismes socio-spatiaux à l'œuvre localement. En effet, au début du $\mathrm{Xx}^{\mathrm{e}}$ siècle, le risque prend d'abord la figure de pollutions atmosphériques caustiques pour les végétaux, représentation véhiculée par un monde rural dominant menacé par la présence non désirée d'une usine chimique de plus en plus exigeante en eau et en hommes. À la fin de ce même siècle, une longue période mono-industrielle a progressivement anéanti la catégorie

12. Cours d'eau de l'Ouest lyonnais.

13. Par le rejet d'eaux savonneuses à même la rivière pour les uns, par l'installation de barrages d'irrigation accentuant les dépôts d'immondices en régime de basses eaux pour les autres. 
des agriculteurs. Et tandis que Rhône-Poulenc entame un processus de désengagement industriel dans l'agglomération, la représentation principale du danger, diffusée par le groupe social devenu dominant des travailleurs de la chimie, s'exprime dans l'agglomération avant tout à travers la crainte du chômage, davantage qu'à travers celles de possibles émanations toxiques et autres explosions (Duchêne, 2001).

À travers ces exemples, nous souhaitons insister sur le fait qu'un groupe social influent localement, selon l'usage qu'il a du sol et plus généralement de la ressource territoriale, se trouve toujours en capacité d'agir sur les modes envisagés de traitement (ou de non-traitement) du danger, en particulier lorsque ceux-ci vont à l'encontre de ses propres intérêts.

\section{2) Risque et valeurs foncières et symboliques}

Un second type de dynamiques territoriales concerne ce qu'il est convenu d'appeler la « valeur» affectée à un quartier, et plus précisément le processus social dans lequel celui-ci se trouve inscrit. En effet, le traitement qui va être appliqué à un territoire précis confronté à un risque donné ne peut être soustrait de ces questions d'ordre plus général. De fait, les réglementations récentes opèrent généralement une modification restrictive sur les droits à construire. Au-delà, elles tendent aussi à désigner des lieux comme étant dangereux, et plus précisément, par le biais de zonages, à distinguer les parcelles soumises à un danger de celles qui ne le seraient pas.

On peut tout d'abord être attentif à la structuration de la propriété dans les zones ainsi désignées. Selon que celle-ci est collective ou individuelle, détenue par des organismes sociaux de type HLM - voire des entreprises logeant leurs salariés ou par des particuliers, les propriétaires affectent à leur patrimoine une valeur qui diffère (en fonction d'attentes spéculatives ou non par exemple), dépassant parfois la seule rationalité économique. Parce qu'elle s'appuie avant tout sur une représentation (tant que la transaction n'est pas effective), la question de la valeur foncière mobilise aussi des registres symboliques (Althabe, Légé et Selim, 1993) : valorisation de son espace de vie, sens que l'on donne à son ancrage territorial ici plutôt qu'ailleurs, ou encore expression d'une forme d'ascension sociale par la propriété, donnant même lieu à l'expression de puissantes formes de valorisation ou de dévalorisation de soi.

Ici ou là, on constate que certains propriétaires concernés tentent de se faire entendre pour que les pouvoirs publics mettent en œuvre des modalités de protection de leur patrimoine sans pour autant encourager la conception de règlements restrictifs stigmatisants ou supposés tels. Ces signes protestataires, lorsqu'ils prennent des formes organisées et récurrentes, révèlent généralement la fragilité de processus sociaux, tant individuels que collectifs, qui animent ces territoires.

Par exemple, «l'association de défense des habitants du Ravin et des zones limitrophes », confrontée, dans l'agglomération lyonnaise, à l'application d'un PPR aux effets restrictifs sur les parcelles riveraines de ce cours d'eau, craignait en apparence une dévalorisation foncière des biens de ses adhérents. Or les faits semblent à 
première vue contredire cette crainte. À l'écoute de ces riverains, propriétaires de pavillons pour l'essentiel, c'est en fait la peur que cette mesure s'accompagne d'un mouvement de paupérisation que ces habitants expriment. Ils évoquent en effet de façon plus sourde, mais récurrente, une zone périurbaine qu'ils considèrent « délaissée », en proie à un morcellement politico-administratif décourageant toute forme d'identification territoriale, et saisie par un envahissement de la circulation automobile au point de remettre en cause le caractère de «zone verte » que l'on prêtait volontiers à cette vallée (Duchêne, Morel Journel et al., 2001).

À l'inverse, pourrait-on dire, l'expression massive d'habitants du quartier des États-Unis (Lyon $8^{\mathrm{e}}$ ), opposés à une nouvelle installation classée dans l'usine chimique riveraine de Givaudan-Roure, a semblé prendre de court les élus locaux, plutôt favorables à la limitation de la désindustrialisation de leur commune comme ils pensaient que les hahitants de ce quartier populaire longtemps ouvrier pouvaient l'être. Or, là encore, à écouter les personnes qui se sont exprimées négativement dans le registre d'enquête publique touchant à l'extension de l'usine, on constate que les habitants reprennent à leur compte le mouvement relatif de « gentryfication » qui saisit leur quartier depuis que les entreprises industrielles qui le composaient ont progressivement fermé leurs portes. Encourager l'extension de Givaudan-Roure, au-delà de son activité dangereuse, pourrait au moins symboliquement entraver la tertiarisation encore fragile de ce quartier lyonnais et ses supposés bienfaits en termes de peuplement et d'image (Mièvre, 2000).

Au moins autant que ce qu'elles semblent dire sur la crainte d'un danger identifié, ces expressions collectives, lorsqu'elles existent, peuvent être aussi entendues comme l'expression plus large d'un processus social, de pauperisation, de « gentryfication » ou d'une autre nature, qui saisit le territoire sur lequel elles s'expriment.

\section{3) Risque et projets d'aménagement urbain}

Enfin un troisième type de dynamique territoriale concerne le développement urbain, considéré comme un processus qui s'épanouit dans le temps. En effet, un territoire existe d'abord au présent, par des pratiques inscrites dans un état matériel palpable ici et maintenant. Pour autant, « pour exister, pour cristalliser l'identité et fabriquer les différenciations socio-spatiales, [il] ne saurait se passer d'une mémoire sociale forgée dans la durée, présente dans les repères patrimoniaux qui le jalonnent et qui le qualifient » (Di Méo, 1998, p. 49). De même, le territoire fait l'objet de projections futures de la part des individus, des groupes sociaux qui l'occupent comme de la part de leurs édiles. Représentations d'un avenir souhaité, discuté, et élaboré pour partie collectivement, les documents d'urbanisme contribuent à ces dynamiques temporelles lourdes, en tant qu'ils tracent dans un moyen terme le cadre du possible en matière de développement.

Chaque projet d'aménagement ou d'équipement, loin de résulter de pratiques spontanées, s'inscrit dans une trajectoire temporelle longue, de son élaboration à son 
mode de financement. Dans cette trajectoire, il est un moment où il « atterrit » et prend place sur une parcelle qui fait elle-même l'objet d'une réglementation prétablie et tout aussi longuement élaborée. Et une incursion « accidentelle » qui viendrait contrecarrer ces processus, telle la définition de zones restreignant les droits à construire, se confronte à la force de ces masses que l'on a lentement mais irrévocablement mises en mouvement.

\section{4) La loi, les réglementations et leurs interprétations locales}

Précisément, lors de la prise en compte des risques au plan local, on constate que l'activité réglementaire se heurte généralement de plein fouet à ces différentes dynamiques territoriales. Véritables artisans de l'articulation entre le global et le local, entre « la norme et le territoire » (Barel, 1981), les élus locaux se trouvent en effet pris entre plusieurs contraintes plus ou moins fortes, plus ou moins prioritaires, et bien souvent contradictoires. En premier lieu, ils sont tenus d'appliquer la loi et de protéger leurs concitoyens. Mais ils ont aussi l'engagement implicite de relayer les intérêts des groupes sociaux qui leur font confiance, la volonté de ne pas dévaloriser les différents territoires qu'ils administrent, ou bien encore l'envie d'inscrire matériellement dans leur commune les projets de développement qui leur sont chers.

Les mesures préventives reflètent généralement les arbitrages adoptés dans ce cadre de préoccupations à bien des égards antagoniques. Les exemples d'élaboration d'une cartographie dessinant les contours de zones de sécurité, destinées à être inscrites dans les plans d'occupation des sols (POS) ${ }^{14}$, autour d'installations chimiques classées de la vallée du Rhône illustrent à la fois la dimension conflictuelle que recèle une législation lorsqu'elle se heurte à un territoire, et la façon dont son application se négocie, prenant en compte l'ensemble des dynamiques qui travaillent ces territoires.

Dans le sud de l'agglomération lyonnaise, à Saint-Fons et Feyzin, communes occupées pour plus du tiers de leur surface par d'importantes installations chimiques et pétrochimiques, le conflit, portant sur l'élaboration de zones dans lesquels il s'agit de geler (Z1) ou de fortement ralentir (Z2) l'urbanisation, est alimenté par au moins deux facteurs. D'une part, la démarche s'inscrit dans une logique initiée et soutenue par l'État, et par lui seulement, alors que le système local d'acteurs concerné par les risques industriels est complexe et morcelé. D'autre part, les enjeux apparaissent très vite considérables, aux yeux des industriels confrontés aux problèmes d'affichage des dangers, comme à ceux des élus locaux, directement concernés par la perspective d'une réduction des possibilités de développement urbain. Entre logiques techniques d'identification et de représentation des dangers, logiques de sécurité et logiques d'aménagement et de développement, il a donc fallu chercher, plusieurs années

14. Devenus depuis plans locaux d'urbanisme (PLU) par l'effet de la loi sur la solidarité et le renouvellement urbain. 
durant, un équilibre subtil, à la fois institutionnel, discursif et réglementaire (Martinais, 1996, 2001).

L'agglomération roussillonnaise a été soumise au même traitement, du fait de la présence d'une importante plate-forme de l'ancien groupe Rhône-Poulenc. Si l'étude de la révision de ses POS dévoile les mêmes formes conflictuelles qu'à SaintFons et Feyzin, la montée des préoccupations judiciaires dans le corps préfectoral au moment de cette élaboration, alliée à un conflit larvé entre les services de la DRIRE et ceux de la $\mathrm{DDE}$, a finalement abouti à une reculade des services préfectoraux vis-àvis de l'accord initial établi avec les collectivités locales à propos de l'emplacement prévu pour un collège d'enseignement. Du coup, la part de négociation qui a prévalu à l'élaboration de ce POS est plus visible encore que dans la périphérie lyonnaise, avec la création d'une Z3 unique en France, dont le règlement consiste, pour caricaturer, à autoriser toute construction... sauf un collège (Duchêne, 1999).

Une large évaluation de l'application locale des lois et réglementations régissant les risques montrerait des situations pour le moins différenciées. Et, pour expliquer ces écarts, au moins au travers des cas déjà étudiés, on voit la nécessité de saisir les processus socio-spatiaux à l'œuvre sur chacun des territoires concernés. Autrement dit, les dispositifs techniques et organisationnels adoptés pour se soustraire au risque ne sont jamais conçus ex nihilo ; ils viennent peu ou prou s'enraciner dans les problématiques structurant la commune où ils s'appliquent.

\section{IV) Risque et intercommunalité : des questions encore largement ouvertes}

Si notre propos a plutôt porté sur l'unité territoriale de base, celle de la commune, il est fréquent que l'échelle à laquelle se manifeste le phénomène redouté ne recoupe pas les échelles territoriales, administratives ou politiques permettant de l'appréhender et de le traiter dans sa globalité. La diversité des enjeux qui se font jour, leur variabilité d'une commune à l'autre, et l'absence d'institution capable de fédérer une réflexion et une action collective, constituent généralement un frein puissant à l'obtention d'une décision consensuelle, satisfaisante pour l'ensemble des protagonistes. Le manque de moyens est un second problème auquel les collectivités locales sont régulièrement confrontées, et ce d'autant plus que le coût des ouvrages de protection dépasse de beaucoup les capacités financières de la commune (ce qui est souvent le cas en milieu rural). Un autre problème, régulièrement évoqué par les responsables des collectivités locales, est leur difficulté à statuer sur des questions exigeant des connaissances particulières dans des domaines de compétence qui leur sont parfois totalement étrangers. Dans leur grande majorité, les élus et les techniciens municipaux ne sont pas des experts et montrent certaines limites lorsqu'il s'agit d'appréhender des phénomènes aussi complexes que ceux dont il est question ici, puis 
de définir les mesures de protection et les dispositifs réglementaires les mieux adaptés aux problèmes posés. Dans des domaines où la science et la technique occupent une place aussi déterminante, où la parole de spécialistes prend autant d'importance, il est manifeste que les représentants des collectivités locales souffrent parfois d'un manque de légitimité qui réduit d'autant leurs capacités d'intervention et leurs marges de manœuvre.

Ces différents points amènent souvent à penser les choses en termes de mutualisation des compétences et des moyens. En effet, dans une vision prospective, et au vu de l'accélération des perspectives intercommunales dans lesquelles les élus locaux sont de plus en plus invités à penser leur action ${ }^{15}$, la modification des échelles territoriales pourrait conduire à repenser les modes locaux de gestion du risque. À l'inverse, compte tenu de l'accumulation des réglementations touchant aux risques ces trente dernières années, ces questions sécuritaires pourraient devenir partie prenante - sinon structurante - dans la recomposition des territoires locaux. Sur ces points, et au vu de l'expérience acquise, sans doute faut-il distinguer risques naturels et technologiques.

Pour les premiers, la réglementation, guidée par les seuls impératifs techniques, a souvent précédé les pratiques politico-administratives. En effet, la loi du 2 février 1995 a institué des plans de prévention des risques (PPR) établis à l'échelle du bassin versant des cours d'eau considérés. Or si cette entité à l'échelle de laquelle se déploient les phénomènes de crues recouvre quelque réalité sur le plan géomorphologique ${ }^{16}$, elle n'a souvent que peu de sens du point de vue des espaces vécus. Imposer comme règle de conduite une solidarité de fait entre territoires amont et aval d'une rivière, en particulier sur les questions touchant à leurs développements futurs, nécessite donc souvent un important travail de construction sociale d'une nouvelle réalité. C'est pour cette raison qu'une vingtaine de communes de l'Ouest lyonnais, concernées à des degrés divers par les débordements récurrents d'un ruisseau à régime torrentiel, a été amenée à se regrouper dans un syndicat de communes destiné à définir et appliquer les mesures de protection ad hoc. Cela étant, plus de dix ans après sa création, aucune réalisation concrète n'a encore été engagée, faute d'avoir encore trouvé les moyens de résoudre à moindre coût leurs divergences quant à l'ouvrage à réaliser et à son mode de financement (Martinais, 1997). En effet, les projets - ou les réticences - de regroupements intercommunaux ne se déploient jamais sur un terrain vierge en ce domaine, tant l'activité des découpages et redécoupages politico-administratifs jalonne 1'histoire de nos territoires administrés (Vant et Gay, 1997). Dans cette perspective, la nouvelle place que la société semble accorder à la question du risque, et en

15. En particulier avec les lois Chevènement « relative au renforcement et à la simplification de la coopération intercommunale » du 12 juillet 1999, Voynet « d'orientation sur l'aménagement et le développement durable du territoire » du 25 juin 1999 et Gayssot « relative à la solidarité et au renouvellement urbain » du 13 décembre 2000 .

16. Encore qu'elle ne prenne pas totalement en compte les écoulements souterrains, partie prenante des phénomènes hydrologiques et qui dessinent parfois d'autres contours que ceux du strict bassin versant (CUDENNEC, 2001). 
corollaire à celle de la sécurité, peut-elle à elle seule créer de nouveaux territoires ? Ou bien le risque ne donnera-t-il lieu en fin de compte qu'à la création d'outils fonctionnels se déployant sur des zones délimitées - et administrées a minima - pour cette seule circonstance? La question semble encore largement ouverte.

S'agissant des risques industriels et de leurs traitements, il est de nouvelles règles dictées par la loi Chevènement qui débouchent sur quelques interrogations. Avec la pratique jusqu'alors en vigueur, via la taxe professionnelle qui leur était versée et dont elles pouvaient fixer les taux, les communes récoltaient ce que l'on pourrait nommer «les bénéfices de la nuisance industrielle». On sait combien ces mécanismes ont été jusqu'à présent puissamment opérants lors de l'installation d'établissements industriels classés, de centrales nucléaires ${ }^{17}$ ou plus récemment de centres d'enfouissement de déchets ultimes. L'institution d'un même taux de taxe professionnelle (la TPU) à l'échelle des structures intercommunales, et surtout l'affectation spécialisée de son produit aux seuls établissements intercommunaux, lorsqu'ils existent, ne vont-elles pas modifier considérablement le regard que portent les élus locaux sur les établissements industriels nuisants qu'ils accueillent dans leur commune ? De même, les industriels concernés peuvent-ils envisager leur activité de la même manière, dans un territoire qui leur serait hostile et auquel ils n'apporteraient pas de compensation immédiate ? Là encore, cette question semble aujourd'hui largement ouverte.

Autrement dit, l'approche intercommunale, si elle apporte des solutions immédiates en termes de mutualisation des moyens et permet de traiter les problèmes à l'échelle spatiale à laquelle ils se posent, ouvre aussi de larges champs d'interrogations qui restent aujourd'hui encore sans réponse simple.

\section{Bibliographie}

G. Althabe, B. LEGÉ et M. Selim, Urbanisme et réhabilitation symbolique - Ivry, Bologne, Amiens, Paris, L'Harmattan, 1993, 297 p.

Y. BAREL, «Modernité, code, territoire», Annales de la recherche urbaine, $\mathrm{n}^{\circ} 10-11,1981$, p. 3-21.

J. Calllosse, « Droit de crise, droit en crise ? ", dans C. Grlbert (dir.), La Catastrophe, l'élu et le préfet, Grenoble, Presses universitaires de Grenoble, 1990, p. 43-66

C. Chaline et J. Dubois-Maury, La Ville et ses dangers. Prévention et gestion des risques naturels, sociaux et technologiques, Paris, Masson, coll. "Pratiques de la géographie », 1994, $247 \mathrm{p}$.

T. CoAnus, F. Duchene et E. MARTinais, La Ville inquiète. Développement urbain, gestion du danger et vie quotidienne sur trois sites «à risque » de la grande région lyonnaise (fin $X I X^{e}-$ fin $\left.X X^{e}\right)$, Laboratoire Rives, ENTPE, 1999, 616 p.

A. Corbin, «L'opinion et la politique face aux nuisances industrielles dans la ville préhaussmannienne », dans Le Temps, le désir et l'horreur. Essais sur le XIX siecle, Paris, Flammarion, coll. « Champs », 1991 (1983), p. 185-198.

17. Sur ce point, voir ZONABEND (1991). 
C. CUDENNEC, « Le bassin versant, territoire structurant les risques hydrologiques », Pré-actes du Colloque international Risques et territoires, UMR-CNRS 5600 Environnement, Ville, Société, 16-18 mai 2001, ENTPE, Vaulx-en-Velin, t. 2, 2001, p. 247-260.

G. DeCrop et C. GLberT, «L'usage des politiques de transition : le cas des risques majeurs », Politique et management public, vol. 11, $\mathrm{n}^{\circ} 2,1993$, p. 143-157.

G. Di MÉo, Géographie sociale et territoires, Paris, Éditions Nathan université, coll. «Fac. géographie », 1998, $320 \mathrm{p}$.

F. Duchêne (dir.), C. Morel Journel (dir.), T. Coanus et E. Martinais, La Culture du risque en question(s) - Etude de deux sites inondables en zone périurbaine de l'agglomération lyonnaise, Laboratoire Rives, ENTPE, 2001, 179 p.

F. DUCHÊNE, Territoires de la chimie. Rhône-Poulenc et la construction de l'agglomération roussillonnaise, thèse pour le doctorat de géographie, (dir.) A. VANT, Université Jean Monnet de Saint-Étienne, 1999, 686 p.

F. DUCHÊNE, « La question du risque industriel, prise entre distance spatiale et distance sociale. Le cas de l'agglomération de Roussillon (Isère) et de "son" usine chimique ", intervention au Colloque international des troisièmes journées de la proximité, Nouvelles Croissances et Territoires, Paris, université Paris X et INRA, 13 et 14 décembre 2001.

C. GILBERT, « Les politiques de traitement des risques naturels et industriels : entre symbolisme et pragmatisme », Annales des Ponts et Chaussées, $\mathrm{n}^{\circ} 76,1985$, p. 11-20

P. LASCOUMES, «La formalisation juridique du risque industriel en matière de protection de l'environnement », Sociologie du travail, $\mathbf{n}^{\circ} 3,1989$, p. 315-333.

C. LEMIEUX et Y. BARTHE, «Les risques collectifs sous le regard des sciences du politique. Nouveaux chantiers, vieilles questions », Politix, $\mathrm{n}^{\circ} 44,1998$, p. 7-28.

J. MARION et X. Prétot, « Les risques naturels et les responsabilités publiques : évolution ou révolution », Administration, $\mathrm{n}^{\circ} 136,1987$, p. $76-81$.

E. Martinais, «Gestion du risque industriel et conflits territoriaux - Le cas de St-Fons, commune de l'agglomération lyonnaise », Revue de géographie de Lyon, vol. $71, \mathrm{n}^{\circ} 1$, 1996, p. 31-44.

E. MARTINAIS et alii, Les Risques d'inondations sur le bassin versant de l'Yzeron. Quelle(s) solution(s) pour une prise en compte globale?, Rapport de diagnostic pour l'Institut des hautes études de la sécurité intérieure, 1997, 29 p. et annexes.

E. MaRTINAIS, Les Sociétés locales à l'épreuve du risque urbain. Un siècle de gestion du danger dans deux contextes de l'agglomération lyonnaise (fin XIX $X^{e}$-fin $\mathrm{XX}^{e}$ siècle), thèse de géographie, université Jean-Monnet de Saint-Étienne, 2001, 665 p.

G. MASSARD-GuILBAUD, «La régulation des nuisances industrielles urbaines (1800-1940) », Vingtieme siècle, revue d'histoire, $\mathrm{n}^{\circ}$ 64, 1999, p. 53-65.

A. MIËVRE, Risque industriel, territoire et information. Le cas de l'usine Givaudan-Roure, quartier des Etats-Unis, Lyon $8^{e}$, mémoire de DEA Villes et sociétés (dir. F. DuCHÊNE), Institut d'urbanisme de Lyon, université Lyon 2, 2000, 142 p. et annexes.

A. VANT et G. GAY, « Saint-Étienne métropole, ou le découpage du territoire minime », Revue de géographie de Lyon, vol. 72, $\mathrm{n}^{\circ} 3,1997$, p. 177-190.

R. VIÉ LE SAGE, La Terre en otage. Gérer les risques naturels majeurs?, Paris, Éd. du Seuil, coll. «Science ouverte », 1989, 248 p.

F. Zonabend, La Presqu'île au nucléaire, Paris, Odile Jacob, 1989, 188 p. 